Musées, Patrimoine et Culture scientifiques et techniques

$144 \mid 2012$

novembre - décembre 2012

\title{
Une réflexion commune et durable sur la mobilisation citoyenne pour la protection de la biodiversité
}

Johanne Landry

\section{OpenEdition}

Journals

Édition électronique

URL : http://journals.openedition.org/ocim/1117

DOI : $10.4000 /$ ocim. 1117

ISSN : 2108-646X

Éditeur

OCIM

Édition imprimée

Date de publication : 1 novembre 2012

Pagination : 5-7

ISSN : 0994-1908

\section{Référence électronique}

Johanne Landry, « Une réflexion commune et durable sur la mobilisation citoyenne pour la protection de la biodiversité », La Lettre de l'OCIM [En ligne], 144 | 2012, mis en ligne le 01 novembre 2014,

consulté le 01 mai 2019. URL : http://journals.openedition.org/ocim/1117 ; DOI : 10.4000/ocim.1117 


\section{Une réflexion}

commune et durable

sur la mobilisation citoyenne pour la protection de la biodiversité

En 2005, Espace pour la vie (Biodôme, Insectarium, Jardin botanique et Planétarium de Montréal) et le Muséum national d'Histoire naturelle de Paris signaient un premier protocole conjoint de collaboration (2005-2010) qui poursuivait plusieurs objectifs : favoriser les échanges entre les membres de leur personnel, développer de nouvelles compétences internes dans différents domaines (conservation, éducation, recherche, diffusion...) et innover au niveau de leur management.

En effet, dans le cadre des réformes vécues par les deux institutions au début des années 2000, les deux muséums souhaitaient développer une plate-forme d'échanges sur la thématique du développement durable et les nouveaux enjeux professionnels de plus en plus importants pour le développement des compétences organisationnelles.

Pour mener cette réflexion, un premier projet intitulé Vers une gestion durable des musées (2007-2009), fut financé conjointement par les deux pays dans le cadre de l'Accord Canada-France. Cette subvention a permis de renforcer les normes professionnelles et les compétences des équipes et a conduit à la création d'un poste de conseiller en développement durable à Espace pour la vie.

Riches des enseignements tirés de ces rencontres et conscients de l'émulation qu'elles provoquent, les deux muséums ont souhaité, pour l'Année internationale de la biodiversité en 2010, profiter de leurs liens privilégiés et poursuivre la réflexion et les échanges de pratiques et d'expertises sur le thème des sciences participatives et la mobilisation citoyenne. Un deuxième projet, Citoyens et biodiversité (2010-2012), initié par Sylvie Tousignant (très engagée dans les dossiers de participation citoyenne à Espace pour la vie), a été proposé à l'Accord Canada-France et a reçu un financement de la part des deux pays contributeurs.

La biodiversité est au cœur des activités d'Espace pour la vie et du Muséum national d'Histoire naturelle. Les différentes équipes de ces deux institutions, que ce soit la recherche, la conservation des collections et la diffusion, œuvrent toutes à la sauvegarde de la biodiversité. Cependant, celle-ci ne pouvant, dans les faits, se réaliser qu’avec le recours de la population, les deux organisations ont proposé de réfléchir sur les moyens dont elles disposent, en tant qu'experts et leaders d'opinion, pour impliquer davantage les citoyens. 
Quatre missions (deux en France et deux au Canada) ont permis aux professionnels des deux institutions muséales, en particulier ceux de la recherche et de la diffusion, de discuter sur les axes de réflexion suivants. Comment sensibiliser les citoyens à la préservation de la biodiversité et à ses enjeux ? Quelles sont les approches et les expériences les plus concluantes? Quels sont les outils les plus performants pour favoriser la participation des citoyens à la protection de la biodiversité ? Comment mobiliser la société civile dans un programme scientifique? Comment utiliser les nouveaux médias sociaux pour rejoindre le plus grand nombre de personnes? Quel est le rôle des associations relais dans la recherche participative comme Vigie-Nature en France ou des projets canadiens comme Monarques sans frontière ou le projet SEM'AIL ?

Il a d'abord fallu développer un corpus de connaissances sur l'approche citoyenne, toujours dans un cadre de développement durable, définir un vocabulaire commun sur les sciences participatives, identifier les méthodes et les outils stratégiques, partager des exemples de mise en œuvre et d'outils de communication à l'interne et l'externe. À la mi-temps du projet, nous avons été associés à une publication de l'Institut de formation et de recherche en éducation à l'environnement (IFRÉE) sur les sciences participatives réalisées par Annie Bauer en 2011 (cette publication intitulée Sciences participatives : portée éducative et pratiques pédagogiques associées est également disponible sur Internet : http://ifree.asso.fr/ UserFiles/Livret_Ifree_n2_Sc-participatives_Coul.pdf).

Ce projet a également impliqué des partenaires universitaires et associatifs : Université du Québec à Montréal (UQAM), Société des Musées québécois (SMQ), Alliance des Musées d'Histoire naturelle du Canada, la Conférence Permanente des Muséums de France, l'Office de Coopération et d'Information Muséales (OCIM), Tela Botanica, Noé conservation, l'OPIE, l'IFRÉE, la LPO... afin de partager cette réflexion avec l'ensemble des acteurs du milieu muséal, universitaire et environnemental.

À l'occasion de la deuxième mission Citoyens et biodiversité à Montréal, l'entente de collaboration entre les deux muséums a été signée pour une deuxième période de 5 ans (2010 2015) entre le directeur général du Muséum national d'Histoire naturelle, Thomas Grenon et le directeur général d'Espace pour la vie, Charles-Mathieu Brunelle. Notons enfin qu'en janvier 2012, un rapport intitulé L'apport des sciences participatives à la connaissance de la biodiversité, rédigé conjointement par Gilles Bœuf, président du Muséum national d'Histoire naturelle, Yves-Marie Allain et Michel Bouvier, a été présenté à la ministre française de l'Écologie, du Développement durable, des Transports et du Logement.

Le projet Citoyens et biodiversité a commencé en mars 2010 et s'est terminé en juin 2012 avec la remise du rapport final aux ministères canadiens et français. Il a permis d'atteindre plusieurs objectifs :

- partager des pratiques innovantes de part et d'autre de l'Atlantique ;

- créer et maintenir des liens privilégiés et durables entre les musées et les professionnels; - améliorer les compétences dans la gestion des fonctions muséales clés, en l'occurrence, la recherche et la diffusion ;

- renforcer les normes professionnelles en termes de sciences participatives et approche citoyenne.

Ce numéro thématique de La Lettre de l'OCIM sur les sciences participatives nous permet de faire une synthèse de cette réflexion amorcée dans le cadre de l'Accord CanadaFrance. Les huit articles proposés présentent divers points de vue qui ont tous été abordés lors de nos rencontres. Tout d'abord, Gilles Bouf nous fait un bilan global des enjeux de sciences participatives, accompagné de la vision d'Espace pour la vie pour reconnecter l'humain à la nature à travers l'article de Charles-Mathieu Brunelle. Daniel Mathieu et Romain Julliard nous présentent deux points de vue, celui d'une association Tela Botanica 
et celui d'une équipe de chercheurs, initiatrice du programme Vigie-Nature. Anne Charpentier, quant à elle, analyse les besoins des jeunes enfants, dans notre société hyper technologique, de jouer dans la nature pour un développement harmonieux ainsi que l'offre des musées dans ce sens. Michèle Lemaire et Sandra Gauthier décrivent ensuite des actions concrètes de sciences participatives dans leurs musées régionaux. Enfin, Michel Venne présente les stratégies de participation citoyenne sur les grands enjeux de société au Québec et à Espace pour la vie.

À travers ce recueil de huit visions convergentes, notre souhait est de susciter une démarche similaire au sein de la communauté muséale francophone afin de favoriser l'émergence de projets novateurs et structurants et créer ainsi un mouvement d'engagement de la population pour une meilleure connaissance et une protection du patrimoine naturel.

Place aux citoyens.

JOHANNE LANDRY

Responsable du projet Grande Place

Espace pour la vie

johanne.landry@ville.montreal.qc.ca 